\title{
Recombinant expression and purification of adenocarcinoma GPR161 receptor
}

\author{
Kasym Kasenovich Mukanov, Zhansaya Batyrbekkyzy Adish, Kanatbek Naizabekovich \\ Mukantayev ${ }^{*}$, Kanat Akhmetovich Tursunov, Zhuldyz Kydyrbekkyzy Kairova, Guldarigash \\ Kuanyshovna Kaukabayeva, Arman Tabylovich Kulyyassov, Pavel Viktorovich Tarlykov
}

National Center for Biotechnology, Kurgali̧byn Road, 13/5, Astana, 010000, Kazakbstan

Received 21st August 2019 / Accepted 3rd October 2019

\begin{abstract}
Triple-negative breast cancer (TNBC) is an aggressive form of breast cancer and very few therapeutic options are currently available for its treatment. Interestingly, G-protein coupled receptor 161 (GPR161) is expressed in TNBC cells and can activate the mammalian target of the rapamycin complex 1 signaling pathway. GPR161 and Ras GTPase-activating-like protein, a protein involved in intracellular signaling, proliferation, and cellular adhesion, have been shown to genetically interact in human breast cancer cells. Targeting of GPR161 by monoclonal antibodies may therefore be a strategy to develop diagnostics and therapeutics for TNBC. Thus, to obtain such monoclonal antibodies, we synthesized the GPR161 gene de novo, cloned it into the pET32 expression plasmid, and used the recombinant plasmid to transform the competent BL21 (DE3) strain of Escherichia coli. The recombinant GPR161 gene was designed to contain an N-terminal thioredoxin tag, a thrombin site, the GPR161 sequence, and a Cterminalhexa-histidine tag to facilitate purification by metal-affinity chromatography. Following purification of the recombinant GPR161 (rGPR161) protein using a HisTrap column, we characterized the protein by Western blotting and mass spectrometry. The rGPR161 protein had a molecular mass of $\sim 49 \mathrm{kDa}$ and its identity as rGPR161 was confirmed by mass spectrometry data using the SwissProt database and the Mascot program. Future studies will involve the development of monoclonal antibodies using rGPR161 as the immunogen.
\end{abstract}

Keywords: Breast cancer, GPR161 receptor, monoclonal antibodies, recombinant protein, tumor diagnostics

\section{INTRODUCTION}

Triple-negative breast cancer (TNBC) accounts for approximately $15 \%$ cancer of the breast diagnosis in women and does not currently have effective targeted therapy. TNBC cells don't express the estrogen (ER), progesterone (PR) and human epidermal growth factor 2 receptor (HER2), and are associated with a poor prognosis. The lack of ER, PR, and HER2 receptors on TNBC tumor cells restricts the use of hormonebased drugs for treatment. However, in breast cancer, there may be different types of cancer that meet the definition of TNBC, for example, basallike breast cancers. This type of breast cancer is characterized by the absence or low levels of expression of ER and low levels of expression of HER2. Both types of cancers (TNBC and basallike breast cancers) are characterized by the presence of a mutation in the $B R C A 1$ gene and a high histological grade (Foulkes et al., 2010; Lachapelle and Foulkes, 2011; Rakha and ReisFilho, 2009).

Breast cancer is generally highly

*Author for correspondence: Dr. Kanatbek Naizabekovich Mukantayev, National Center for Biotechnology, Kurgalzhyn Road, 13/5, Astana, 010000 Kazakhstan. Email - mukantaev@biocenter.kz 
heterogeneous and the different types are identified by their histopathological features, genetic alterations, and gene-expression profiles (Sotiriou and Pusztail, 2009). Kreike et al. (2007) characterized TNBC at the gene expression and histopathological levels. Based on their gene expression profiling study, they found that TNBC is itself heterogeneous and can be subdivided into at least five distinct subgroups. Because of the high levels of genetic heterogeneity, TNBC lacks both effective targeted therapies and diagnostics.

An important goal in studying triple-negative tumors is to identify novel therapeutic and diagnostic targets within this group of tumors. This is especially important as these tumors do not respond to ER- and HER2-targeted therapies since $73 \%$ of TNBC tumors are epidermal growth factor receptor (EGFR)-negative. Haffty et al. (2006) used simple commonly available markers for ER, PR, and HER2/neu and found that patients with TNBC have a relatively poor prognosis. Since they lack ER, PR, and HER2/neu receptors, patients with triplenegative breast cancer are not candidates for adjuvant hormonal therapy or trastuzumab.

$G$ protein-coupled receptors (GPCRs) mediate different physiological processes and introduce the targets for a broad array of drugs for different diseases. GPCRs transduce extracellular signals from a variety of ligands through the activation of heterotrimeric G-proteins and downstream second messengers. Through this mechanism, rapid signaling events occur via the formation of short-lived secondary signals. GPCRs can also act as regulators of oncogenesis. Transactivation of signaling molecules, including EGFR, occurs by this mechanism. Because they can be activated by a wide variety of ligands, GPCRs play important roles in the pathogenesis of hormone-responsive tumors. For example, somatic mutations found in the thyrotropin receptor result in the activation of adenylyl cyclase, leading to hyperactivation of thyroid adenomas. Thus, initiating mutations have been found in the luteinizing hormone receptor in Leydig-cell testicular tumors. Some new studies have also demonstrated the role of GPCRs in regulating cancer cell invasion and metastasis (Feigin, 2013).

Using large-scale genomic analysis, Feigin $e t a l$. (2014) discovered a poorly characterized receptor referred to as GPR161 that was up-regulated in TNBC. The GPR161 receptor was characterized as a prognostic biomarker for TNBC and was found to regulate the proliferation and migration of breast cancer cells. The authors also demonstrated the importance of GPR161 in the pathogenesis of TNBC by providing evidence that GPR161 promotes proliferation by activating mTORC1. The GPR161 receptor also regulates migration and invasion by disrupting the localization of E-cadherin (E-cad). The authors also demonstrated that GPR161 induces proliferation and migration in an IQGAP1dependent manner. On the basis of these data, GPR161 appears to promote cancer cell proliferation and migration and is a promising drug target for the treatment of TNBC.

\section{MATERIALS AND METHODS}

\section{Bacterial strain, plasmids, and antibodies}

E. coli DH5 $\alpha$ and BL21 (DE3) (Novagen, Madison, WI, USA), and plasmids pET28 and pET32 (Novagen) were used in this study. All E. coli strains were cultured in lysogeny broth (LB) medium. An anti-His-tag mouse monoclonal antibody (Sigma- Aldrich, Taufkirchen, Germany) and a peroxidase-conjugated secondary antibody (Sigma-Aldrich) were used for Western blotting.

\section{Gene synthesis}

The amino acid sequence of the GPR161 receptor isoform 1 (Homo sapiens) was extracted from PubMed with the NCBI sequence reference NP_001254538.1. Part of the sequence of the 7tm_GPCR (seven-transmembrane GPCR) encompassing amino acids 64-344 was selected for further study. The gene sequence was codonoptimized for expression in E. coli using Vector NTI 11.5 software, and the GPR161 receptor cDNA was synthesized by Macrogen Inc., Korea. The synthetic GPR161 cDNA was cloned into pET28 and pET32 plasmids using the NcoI and XhoI restriction sites so that the final pET32 plasmid construct contained an N-terminal thioredoxin tag, a thrombin site, the GPR161 receptor, and six C-terminal His-tags. The final pET28-based construct contained the same sequences except for the $\mathrm{N}$-terminal thioredoxin 
tag. The predicted molecular weights of the Histagged recombinant GPR161 in pET32 and pET28 were $\sim 49 \mathrm{kDa}$ and $\sim 36 \mathrm{kDa}$, respectively.

\section{Transformation of $E$. coli and expression of rGPR161}

Competent BL21 (DE3) E. coli were transformed with the pET28 and pET32 plasmid vectors, with or without the gene insert, by electroporation using a MicroPulser (Bio-Rad, Hercules, CA, USA) under the following conditions: $100 \mathrm{ng}$ of plasmid per $50 \mu \mathrm{L}$ of cell suspension, at $2.5 \mathrm{kV}$, $25 \mu \mathrm{F}$, and $200 \Omega$. Electroporation duration was $5.2 \mathrm{~ms}$. The transformed cells were incubated in $950 \mu \mathrm{L}$ of superoptimal broth at $37^{\circ} \mathrm{C}$ for $1 \mathrm{~h}$ with rotary shaking at $200 \mathrm{rpm}$. Following this, $50 \mu \mathrm{L}$ of cells were seeded onto LB agar containing ampicillin as the selection antibiotic and grown at $37^{\circ} \mathrm{C}$ for $16 \mathrm{~h}$. Single colonies of transformants were cultured in $2 \times \mathrm{YT}$ broth containing ampicillin. In the middle of the logarithmic growth phase of the bacterial mass $\left(\mathrm{OD}_{600}=0.6\right)$, $0.1 \mathrm{mmol} / \mathrm{L}$ of the inducer, isopropyl- $\beta-\mathrm{D}-1-$ galactopyranoside (IPTG), was added and the culture incubated for $6 \mathrm{~h}$ at $26^{\circ} \mathrm{C}$. Cells were then pelleted by centrifugation at $6,000 \times g$ for $7 \mathrm{~min}$ at $4^{\circ} \mathrm{C}$. For sequencing, the GPR 161 gene product was transformed into the DH5 $\alpha$ E. coli strain. E. coli colonies were grown on solid agar medium and analyzed by PCR using Taq polymerase and M13 primers. Four positive clones were used for DNA purification and sequencing using the BigDye Terminator reagent kit (Thermo Fisher Scientific, Austin, TX,USA)

\section{Cell lysis and chromatographic purification}

For purification of the recombinant receptor, we used a protocol based on that reported by Attrill et al. (2009). Cells were lysed in an ice-cold buffer (20 mmol/L NaCl, $20 \mathrm{mmol} / \mathrm{L}$ HEPES, 0.1 $\mathrm{mmol} / \mathrm{L}$ phenylmethylsulfonyl fluoride (PMSF), and $0.5 \%$ CHAPS, $\mathrm{pH} 7.5$ ) using a UP200S ultrasonic disintegrator set at $24 \mathrm{kHz}$ in a pulsating mode (10 pulses, $10 \mathrm{~s}$ per pulse). Cell debris was pelleted at $60,000 \times g$ for $1 \mathrm{~h}$. The detergent-solubilized fraction was filtered through a $0.2-\mu \mathrm{m}$ syringe filter (Millipore, Burlington, MA, USA). Recombinant protein was purified using metal-chelate chromatography on $\mathrm{Ni}^{2+}$ ions using a $1-\mathrm{mL}$ HisTrapTM HP column (GE Healthcare, Piscataway, NJ, USA).
The protein solution was placed onto the $\mathrm{Ni}^{2+}$ NTA column ( $2 \mathrm{~mL}$ bed volume) equilibrated with the same buffer. The column was washed with 10-bed volumes of the equilibration buffer (20 mmol/L Tris-HCl, $\mathrm{pH}$ 8.0, containing 500 $\mathrm{mmol} / \mathrm{L} \mathrm{NaCl}, 50 \mathrm{mmol} / \mathrm{L}$ imidazole, $0.5 \%$ CHAPS, and 0.1 PMSF). The GPR161 protein was then eluted with a $20-500 \mathrm{mmol} / \mathrm{L}$ linear imidazole gradient. The protein concentrations in the cell lysate and purified fractions were determined using the Bradford assay with bovine serum albumin as the standard (Bradford, 1976).

\section{Western blotting}

Fractions containing the GPR161 receptor were separated by electrophoresis on $11 \%$ polyacrylamide gels containing sodium dodecyl sulfate (SDS) according to the Laemmli method using a Bio-Rad electrophoresis apparatus (BioRad) (Laemmli, 1970). Proteins were electrophoretically transferred onto nitrocellulose membranes using an immunoblotting device (BioRad) according to a previously published method (Towbin et al., 1979).

For immunochemical detection, the nitrocellulose membranes were first incubated in a blocking solution (phosphate-buffered saline (PBS), $\mathrm{pH} 7.4$, containing 1\% bovine serum albumin) overnight at $4{ }^{\circ} \mathrm{C}$ and then washed three times in PBS, pH 7.4, containing $0.05 \%$ Tween20 (PBST). Membranes were incubated for $1.5 \mathrm{~h}$ at $37^{\circ} \mathrm{C}$ in a blocking buffer containing a 1:2000 dilution of a mouse monoclonal antibody against the His-tag. Subsequently, the membranes were rewashed as above and incubated in a blocking buffer with peroxidase-conjugated secondary antibody at 1:10000 dilution for $1 \mathrm{~h}$ at $37^{\circ} \mathrm{C}$. The substrate solution was prepared immediately before use as follows: $0.01 \mathrm{~g}$ of 4-chloro-naphthol (Sigma, St. Louis, MO, USA) was dissolved in 2 $\mathrm{mL}$ of methanol, and mixed with $18 \mathrm{~mL}$ of buffer 1; finally, $0.01 \mathrm{~mL}$ of $3 \%(\mathrm{v} / \mathrm{v})$ hydrogen peroxide was added. This substrate solution was applied to the blots for visualization of immunoreactive protein bands. The blot was placed in the substrate solution and incubated for $15 \mathrm{~min}$ at room temperature until the stained bands appeared. 


\section{Nano liquid chromatography and tandem mass spectrometry (nanoLC-MS/MS)}

Workbench surfaces were routinely cleaned of dust using a clean, damp paper towel to avoid sample contamination with keratin. To ensure sample purity, we also used keratin-free Eppendorf tubes and barrier tips during sample preparation. Purified GPR161 samples were fractionated by $11 \%$ SDS- polyacrylamide gel electrophoresis (PAGE) and the gels were stained with Coomassie blue. The protein bands were precisely excised and transferred to keratin-free Eppendorf tubes. The excised bands were then divided into small gel fragments of $1 \times 1 \mathrm{~mm}$ dimension. To destain the gel pieces of Coomassie blue, $100 \mu \mathrm{L}$ of $100 \mathrm{mM}$ ammonium bicarbonate in acetonitrile $(1: 1)$ was added and the gel fragments were incubated at $37^{\circ} \mathrm{C}$ for $30-40$ min. After removing the supernatant, $5 \mathrm{mM}$ dithiothreitol (DTT) was added to each tube and incubated at $60^{\circ} \mathrm{C}$ for $10 \mathrm{~min}$. The DT'T solution was then removed and $100 \mu \mathrm{L}$ of $100 \mathrm{mM}$ iodoacetamide was added and incubated at $37^{\circ} \mathrm{C}$ for $15 \mathrm{~min}$ to alkylate the reduced cysteine residues in the proteins. Excess reagent was then removed, and the gel pieces were washed twice in $100 \mu \mathrm{L}$ of $50 \mathrm{mM}$ ammonium bicarbonate. To remove any residual iodoacetamide, the gel pieces were subjected to two cycles of dehydration in 200 $\mu \mathrm{L}$ of $100 \%$ acetonitrile and rehydrated in $50 \mathrm{mM}$ ammonium bicarbonate in water. The gel pieces were then dehydrated in 100\% acetonitrile for 35 min to reduce their size, after which the acetonitrile was removed and the tubes dried for $5 \mathrm{~min}$. Finally, $2 \mu \mathrm{L}$ of $100 \mathrm{ng} / \mu \mathrm{L}$ trypsin and 50 $\mu \mathrm{L}$ of $50 \mathrm{mM}$ ammonium bicarbonate were added, and the tubes were incubated overnight at $37^{\circ} \mathrm{C}$ to allow trypsin digestion to occur. On the following day, the supernatant containing the peptide mixtures resulting from trypsin digestion were transferred to clean Eppendorf tubes. For the second extraction of the digested peptides, the remaining gel pieces were washed in $50 \mu \mathrm{L}$ of 50 $\mathrm{mM}$ ammonium bicarbonate, incubated for 15-20 min, and the supernatant transferred into the same tube containing the peptide mixture from the previous extraction. The contents of the tubes were dried using a vacuum concentrator at $45^{\circ} \mathrm{C}$ for 30-60 min. After complete removal of water, the resultant residue (containing the tryptic peptides) was dissolved in $10 \mu \mathrm{L}$ of $0.1 \%$ trifluoroacetic acid, and the soluble peptide mixture was desalted using a Zip-tip kit (Millipore ZiptipsMicro-C18, $0.2 \mu \mathrm{L}$,Sigma).

The resulting mixture of tryptic peptides was separated using high-performance liquid chromatography (HPLC) and analyzed by in-line MS/MS. For LC-MS/MS, an Acclaim ${ }^{\mathrm{TM}}$ PepMap ${ }^{\text {TM }} 100$ C18 pre-column $(5 \mathrm{~mm} \times 300 \mathrm{~cm}$; $5 \mu \mathrm{m}$ particle size; Thermo Fisher Scientific) was used with a Dionex HPLC pump (Ultimate 3000 RSLC nano System, Thermo Fisher Scientific). The peptide mixture was separated on an Acclaim $^{\text {TM }}$ Pep-Map ${ }^{\text {TM }}$ RSLC column $(15 \mathrm{~cm} \times$ $75 \mu \mathrm{m}, 2 \mu \mathrm{m}$ particle size; Thermo Fisher Scientific) using a 75-min multistage acetonitrile gradient (buffer A, 0.1\% formic acid; buffer B, $90 \%$ acetonitrile $/ 10 \% \mathrm{H}_{2} \mathrm{O}$ in $0.1 \%$ formic acid) at a flow rate of $0.3 \mu \mathrm{L} / \mathrm{min}$. The gradient program for buffer B was as follows: $0 \mathrm{~min}-2 \%, 10 \mathrm{~min}-$ $2 \%, 58 \min -50 \%, 59 \min -99 \%, 69 \min -99 \%$, $70 \mathrm{~min}-2.0 \%, 75 \mathrm{~min}-2.0 \%$. The unmodified CaptiveSpray ion source (Capillary $1300 \mathrm{~V}$, dry gas $3.0 \mathrm{~L} / \mathrm{min}$, dry temperature $150^{\circ} \mathrm{C}$ ) was used to interface the chromatography system with the Impact II (Bruker, Billerica, MA, USA). Subjecting the mixture of digested peptides to chromatography ensured the removal of lowmolecular-weight impurities. The tandem MS/MS conditions were as follows: two of the most intense precursor ions to obtain sample data were selected for subsequent fragmentation with a fulltime cycle of $3 \mathrm{~s}$. The mass range was from 150 to $2,200 \mathrm{~m} / \mathrm{z}$ under the positive ion mode.

The Mascot software was used to search the SwissProt 2016_10 database (552,884 sequences; 197,760,918 residues). Search parameters included variable modifications, including cysteine carbamidomethylation and methionine oxidation, a fragment ion mass tolerance of 0.6 $\mathrm{Da}$, and a mass tolerance of the parent ion of 1.20 Da.

\section{RESULTS}

\section{Design and construction of the expression vector}

Comparison of the amino acid sequence of the GPR161 receptor isoform 1 with all GPR161 sequences present in the NCBI database revealed 
that the GPR161 isoform 1 receptor is homologous to other members of this family of receptors (Figure 1). The codon-optimized nucleotide sequence of the selected region (length $897 \mathrm{bp}$ including the restriction sites) (Figure 2) was inserted into the pET28 and pET32plasmids. Then, $\mathrm{DH} 5 \alpha$ bacteria transformed with the vector containing the correct insert were identified and further confirmed by DNA sequencing to contain the insert encoding the fragment of recombinant transmembrane receptor

GPR161

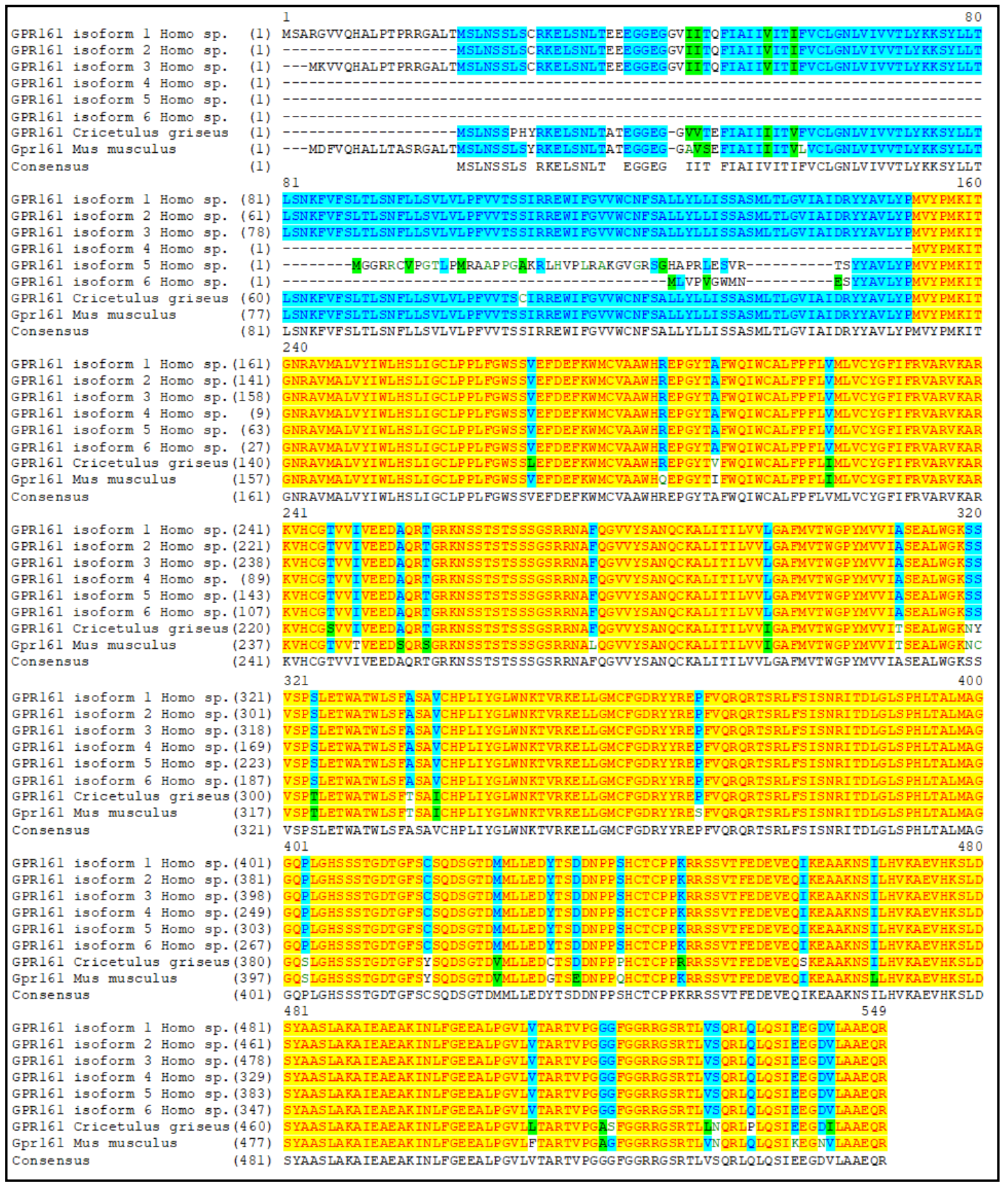

Figure 1. Homology of the GPR161 receptor isoform 1 with other members of the GPR161 family. Yellow color - the complete match of the nucleotide sequence of all receptor isoforms; blue and green colors - variations of nucleotide sequences. 


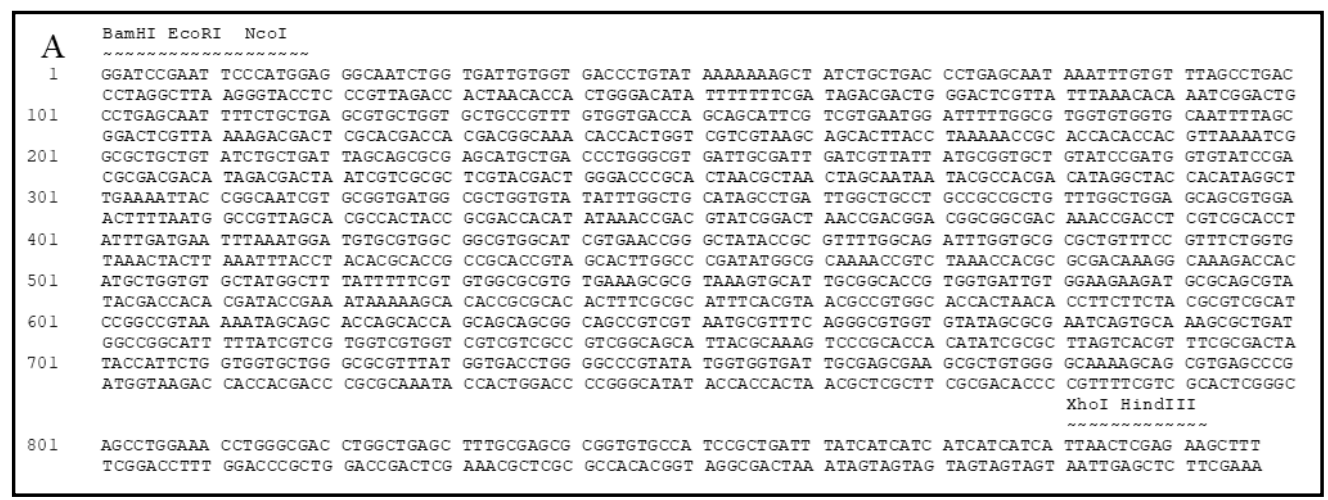

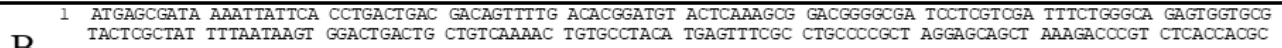 B ClaI
101 GTCCGTGCAA AATGATCGCC OCGATTCTGG ATGAAATCGC TGACGAATAT CAGGGCAAAC TGACCGTTGC AAAACTGAAC ATCGATCAAA ACCCTGGCAC GTCGGGCAA AATGATCGCC OCGATTCTGG ATGAAATCGC TGACGAATAT CAGGGCAAAC TGACCGTTGC AAAACTGAAC ATCGATCAAA ACCCTGGCAC
CAGGCACGTT TTACTAGCGG GGCTAAGACC TACTTTAGCG ACTGCTTATA GTCOCGTTTG ACTGGCAACG TTTTGACTTG TAGCTAGTTI TGGGACCGTG ApaLI
201 TGCGCCGAAA TATGGCATCC GTGGTATCCC GACTCTGCTG CTGTTCAAAA ACGGTGAAGT GGCGGCAACC AAAGTGGGTG CACTGTCTAA AGGTCAGTTG TIGGG CTGAGACGAC GACAAGTTTT TGCCACTTCA CCGCCGTTGG ITTCACCCAC GTGACAGATT TCCAGTCAAC
301 AAAGAGTCC ICGACGCIAA CCTGGCDGGT TCTGGTTCTG GCCATATGCA CCATCATCAT CATCATTCTT CTGGTCTGGT GCCACGCGGT TCTGGTATGA TITCTCAAG AGCTGCGATT GGACCGGCCA AGACCAAGA CGGTATACGT GGTAGTAGTA GTAGTAAGAA GACCAGACCA CGGTGCGCCA AGACCATACT NCoI
401 AAGAAACCGC TGCTGCTAAA TTCGAACGCC AGCACATGGA CAGCCCAGAT CTGGGTACCG ACGACGACGA CAAGGCCATG GAGGGCAATC TGGTGATTGT 1 GGTGACCCTG tataAaAaAa GCTATCTGCT GaCCCTGAGC AATAAATTTG TGTTTAGCCT GACCCTGAGC AatTTTCTGC TGAGCGTGCT GGTGCTGCCG CCACTGGGAC ATATTTTTTI CGATAGACGA CTGGGACTCG TTATTTAAAC ACAAATCGGA CTGGGACTCG TTAAAAGACG ACTCGCACGA CCACGACGGC
601 TTTGTGGTGA CCAGCAGCAT TCGTCGTGAA TGGATTTTTG GCGTGGTGTG GTGCAATTTT AGCGCGCTGC TGTATCTGCT GATTAGCAGC GCGAGCATGC AAACACCACT GGTCGTCGTA AGCAGCACTT ACCTAAAAAC CGCACCACAC CACGTTAAAA TCGCGCGACG ACATAGACGA CTAATCGTCG CGCTCGTACG ACTGGACCC GCACAACGC IAACTGGCTG CCTGCCGCCG CTGTTTGGCT GGAGCAGCGT GGAATTTGAT GAATTTAAAT GCACGCCACT ACCGCGACCA CATATAAACC GACGTATCGG ACTAACCGAC GGACGGCGGC GACAAACCGA CCTCGTCGCA CCTTAAACTA CTTAAATTTA CCTACACGCA CCGCCGCACC 901 CATCGTGAAC CGGGCTATAC CGCGTTTTGG CAGATTTGGT GCGCGCTGTT TCCGTTTCTG GTGATGCTGG TGTGCTATGG CTTTATTTTT CGTGTGGCGC GTAGCACTTG GCCCGATATG GCGCAAAACC GTCTAAACCA CGCGCGACAA AGGCAAAGAC CACTACGACC ACACGATACC GAAATAAAAA GCACACCGCG 001 GTGTGAAAGC GCGTAAAGTG CATTGCGGCA CCGTGGTGAT TGIGGAAGAA GATGCGCAGC GTACCGGCCG TAAAAATAGC AGCACCAGCA CCAGCAGCAG CACACTTTCG CGCATITCAC GIAACGCCGT GGCACCACTA ACACCTTCTI CTACGCGICG CATGGCCGGC ATTTTTATCG TCGTGGTCGT GGTCGTCGTC 1101 CGGCAGCCGT CGTAATGCGT TTCAGGGCGT GGTGTATAGC GCGAATCAGT GCAAAGCGCT GATTACCATT CTGGTGGTGC TGGGCGCGTT TATGGTGACC 1 TGGGGCCCGT DTDTGGTGGT GATTGCGAC GDAGCGCTGT GGGGCDAAAG CAGCGTGAGC CCGAGCCTGG AAACCTGGGC GACCTGGCTG AGCTTTTGCGA ACCCCGGGCA TATACCACCA CTAACGCTCG CTTCGCGACA CCCCGITTTC GTCGCACTCG GGCTCGGACC TITGGACCCG GTGGACCGAC TCGAAACGCT XhoI
1301 GCGCGGTGTG CCATCCGCTG ATTTATCATC ATCATCATCA TCATTAACTC GAGAATCAC

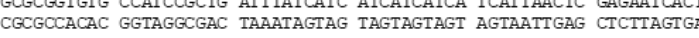

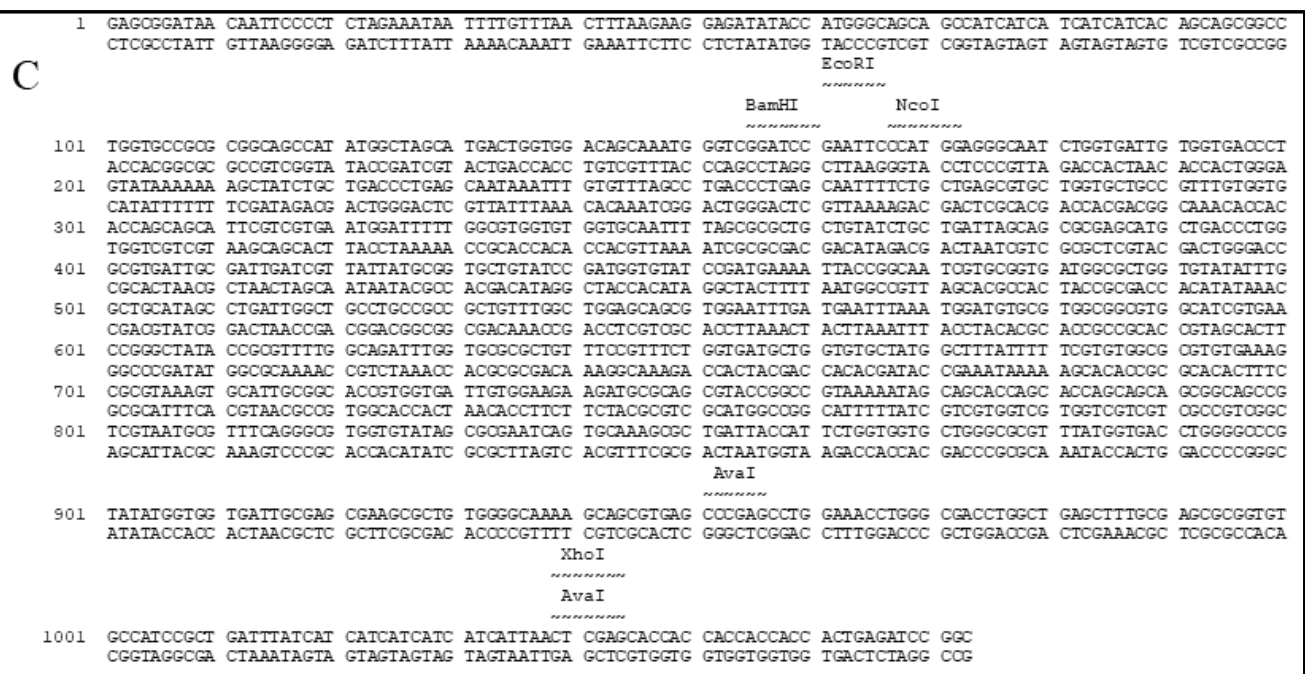

Figure 2. Nucleotide sequence of the codon-optimized GPR161 fragment encoding amino acids 64-344 (rTMGPR161) (A), nucleotide sequence of the codon-optimized GPR161 fragment cloned into the pET32 plasmid (B) and nucleotide sequence of the codon-optimized GPR161 fragment cloned into the pET28 plasmid (C). 


\section{Transformation with the gene construct and establishment of an $E$. coli strain producing rTMGPR161}

The expression vectors pET28/rTMGPR161 and pET32/rTMGPR161 were transformed into $E$. coli BL21. The strains obtained were then analyzed for the expression of the recombinant GPR161 protein. To detect protein expression, E. coli strains were cultured in LB medium supplemented with $0.2 \mathrm{mM} \mathrm{IPTG}$ at $26^{\circ} \mathrm{C}$. After the addition of IPTG, samples of the E. coli culture were taken at different time points $(2,4,6$, and $24 \mathrm{~h}$ ), sonicated, lysed, and subjected to SDSPAGE followed by Coomassie blue staining. There was no recombinant protein expression in BL21 E. coli transformed with pET28/rTMGPR161(Figure 3(A)). In contrast, there was expression of rTMGPR161 in BL21 E. coli transformed with pET32/rTMGPR161 (Figure 3(B)). The rTMGPR161 protein was expressed $2 \mathrm{~h}$ after IPTG addition and had an approximate molecular mass of $\sim 49 \mathrm{kDa}$.
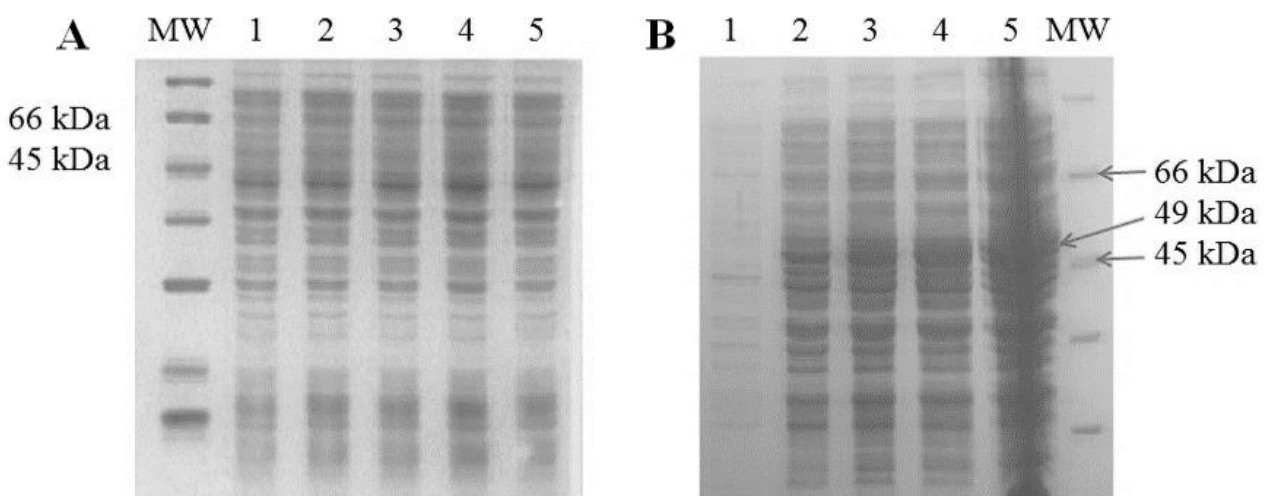

Figure 3. SDS-PAGE of total proteins expressed in the strains BL21/pET28/rTMGPR161 (A) and BL21/pET32/rTMGPR161 (B). Lane 1 - E. coli culture without IPTG; Lane 2 - E. coli culture $2 \mathrm{~h}$ after IPTG addition; Lane 3 - E. coli culture $4 \mathrm{~h}$ after IPTG addition; Lane 4 - E. coli culture after $6 \mathrm{~h}$ incubation with IPTG; Lane 5 - E. coli culture after 24 h incubation with IPTG; MW - molecular weight markers.

\section{Isolation and purification of rTMGPR161}

A representative SDS-PAGE analysis of soluble and insoluble fractions from BL21/pET32/rTMGPR161 is shown in Figure 4. Following cell lysis and centrifugation to remove insoluble debris, the rTMGPR161 fusion protein remained present in the soluble fraction (Figure 4(A)) of the cell lysate, while it was not detectable in the pellet (Figure 4(B)).

To optimize the isolation and purification of rTMGPR161, the BL21/pET32/rTMGPR161 E. coli were cultured in $2 \times$ YT medium containing ampicillin and various IPTG concentrations $(0.05$ $\mathrm{mM}, 0.1 \mathrm{mM}, 0.2 \mathrm{mM}$, and $0.4 \mathrm{mM}$ ), at different time points (2, 4, 6, and $24 \mathrm{~h})$ and at a temperature of $26^{\circ} \mathrm{C}$. We found that the conditions for the optimal expression of rTMGPR161 were media containing $0.2 \mathrm{mM}$ IPTG and $24 \mathrm{~h}$ incubation (Figure 5).

After purification of the rTMGPR161 protein by $\mathrm{Ni}^{2+}$-Sepharose chromatography and elution using a buffer containing $200 \mathrm{mM}$ imidazole, we used SDS-PAGE and Coomassie blue staining to visualize the purified rTMGPR161 protein. As can be seen in Figure 6(A), chromatography yielded fraction containing purified rTMGPR161 with an expected molecular weight of about 49 $\mathrm{kDa}$. To confirm expression of the correct protein, we used an anti-His-tag monoclonal antibody in Western blotting of the separated by SDS-PAGE rTMGPR161 samples (Figure 6(B)). This Western blotting analysis confirmed the presence of the hexahistidine tag on a protein with a molecular mass of $\sim 49 \mathrm{kDa}$, which corresponds to the predicted molecular mass of rTMGPR161.

\section{LC-MS/MS analysis of $r$ TMGPR161}

LC-MS/MS was used to confirm the identity of rTMGPR161. The MS/MS spectra of peaks corresponding to the fragmented ions of peptides derived from trypsin-digested rTMGPR161 were identified following SDS-PAGE, trypsin 
digestion, and chromatographic separation. Trypsin-digested peptides are characterized by the presence of lysine or arginine residues at the Cterminus of the peptide. The MS/MS spectra were converted to mgf files using the DataAnalysis program. These files were submitted to the Mascot search engine, which compares the experimental data with theoretical mass spectra using available sequence databases of amino acids, such as NCBI or SwissProt.

The score (86.8) corresponded to only one protein, namely GPR161. Representative MS/MS spectra of the EGNLVIVVTLYKKSYLLT LSNKF peptide of rGPR161 and its fragmentation ions are shown in Figure 7.

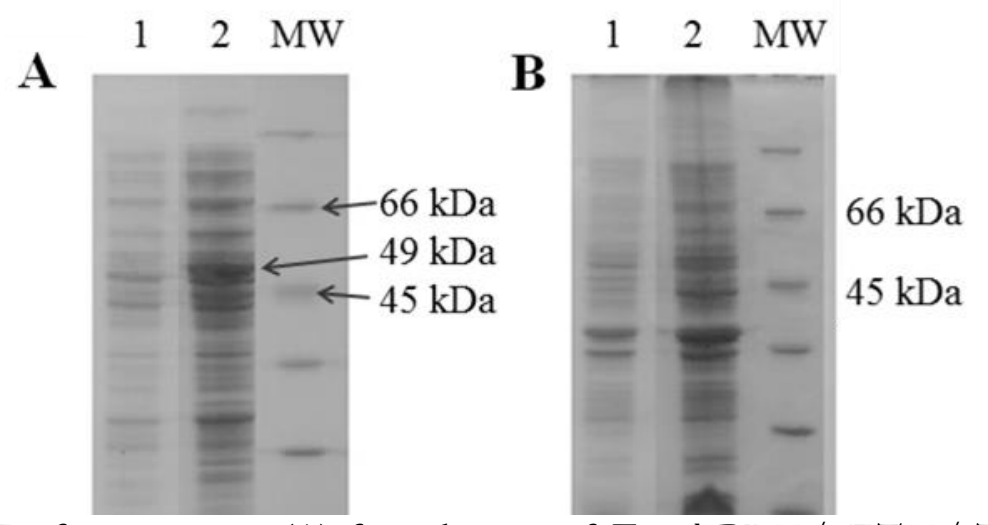

Figure 4. SDS-PAGE of supernatant (A) from lysates of E. coli BL21/pET32/rTMGPR161and pellet (B). Lane 1 - E. coli culture without IPTG; Lane 2 - E. coli culture after 24 h incubation with IPTG; MW molecular weight markers.

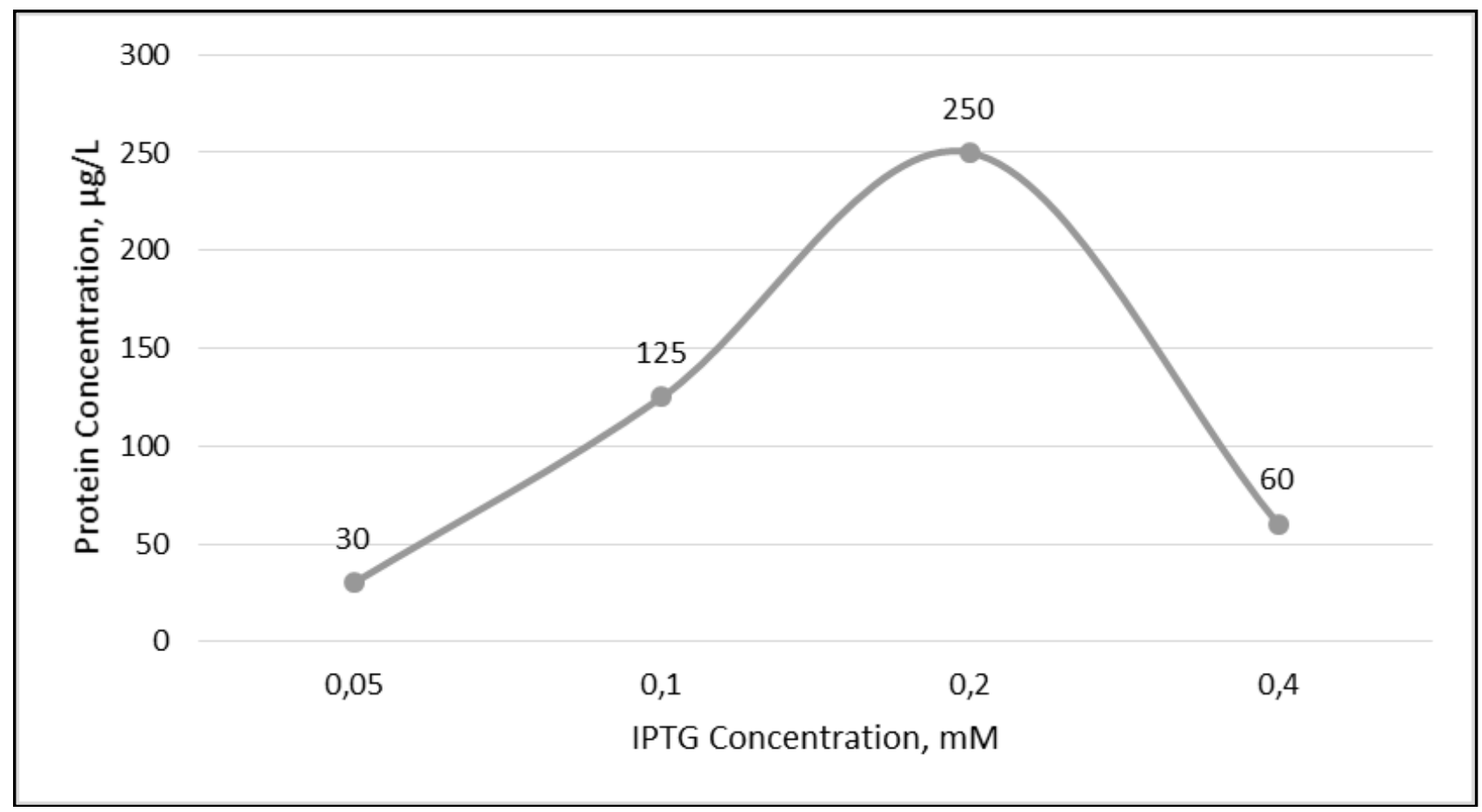

Figure 5. Graph of rTMGPR161 concentration versus IPTG concentration. 


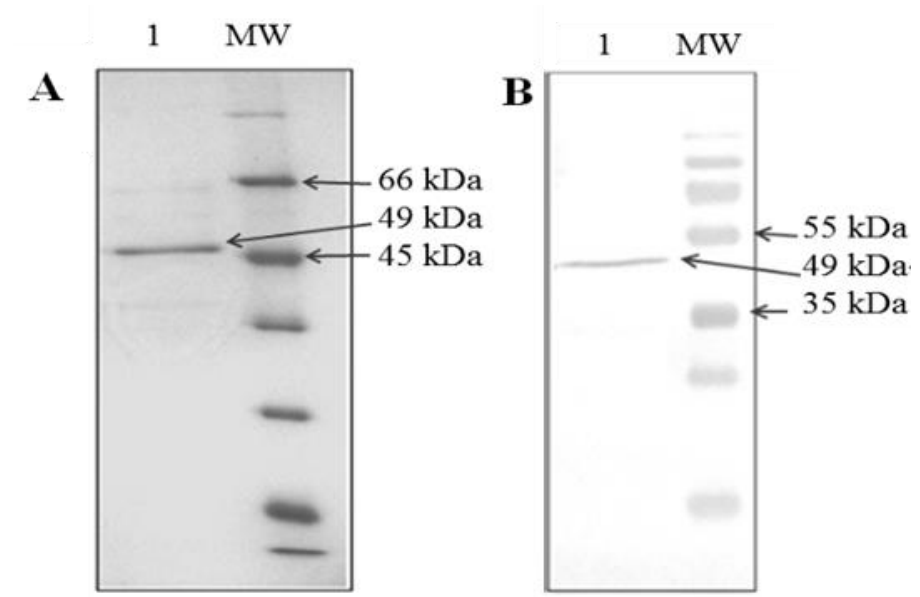

Figure 6. SDS-PAGE (A) and Western blot (B) of purified proteins expressed and extracted from the BL21/pET32/rTMGPR161 E. coli. Lane 1 - purified rTMGPR161; MW - molecular weight markers.

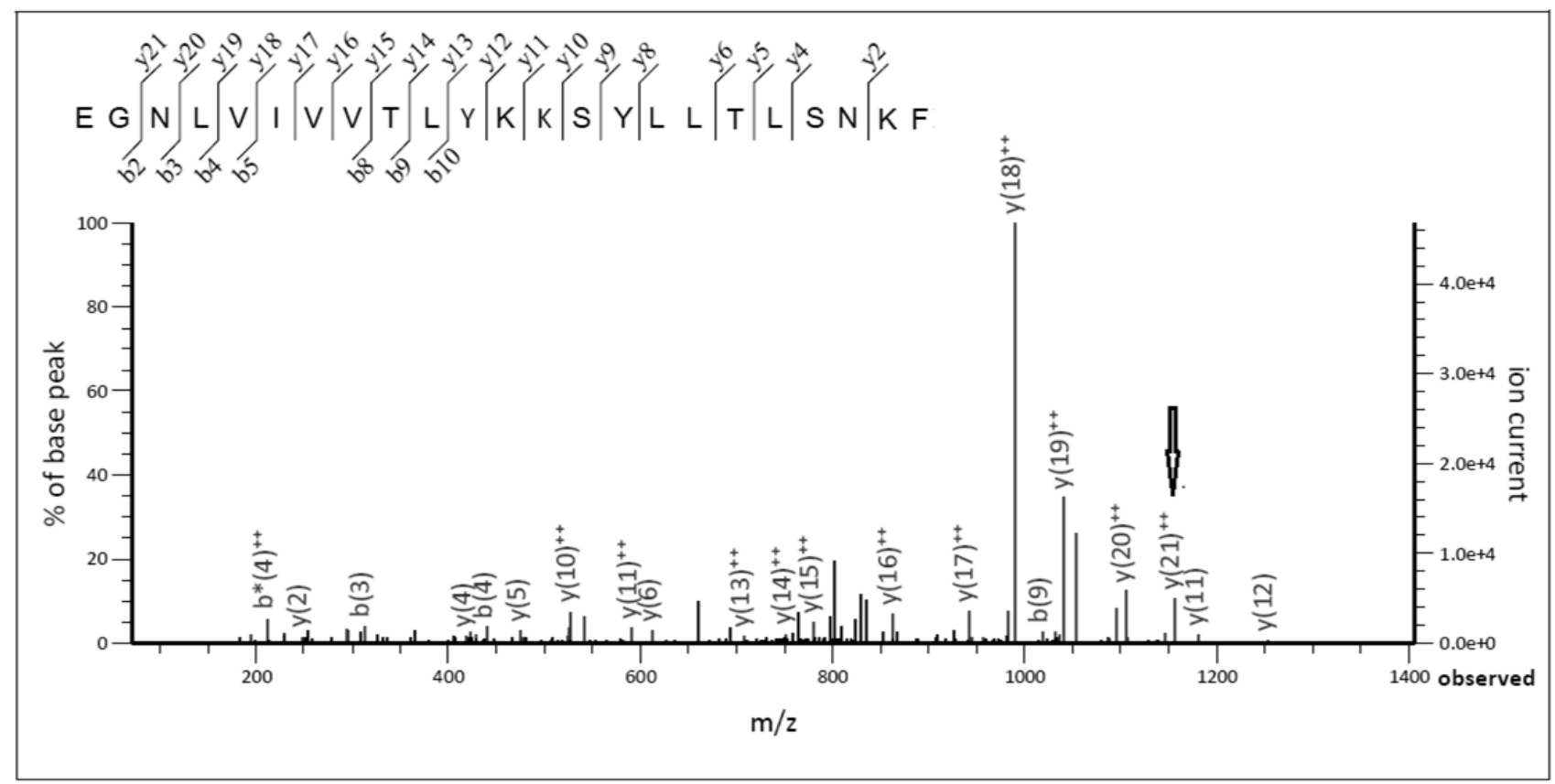

Figure 7. MS/MS spectra of fragmented peptides derived from trypsin-digested rTMGPR161.

\section{DISCUSSION}

The GPR161 receptor is a prognostic biomarker of TNBC and an important regulator of the proliferation and migration of breast cancer cells. The GPR161 receptor decreases IQGAP1 serine phosphorylation and activates the mTOR/p70S6K signaling pathway. Genetic analyses of the GPR161 receptor in TNBC cells have identified two mutations. The first mutation (R91G) is located within the first extracellular loop and may, therefore, play a role in ligand coupling. The second mutation, S251G, is found within the third intracellular loop, a region which is phosphorylated in response to the activation of many GPCRs and is the site of interaction of $\beta$ arrestins (Feigin et al., 2014). Genetic analyses of other cancer types have uncovered their current amplification of the GPR161 gene in bladder urothelial carcinoma, lung adenocarcinoma, and melanoma. It is therefore likely that developing methods to target GPR161 may have diagnostic and therapeutic applications beyond breast cancer.

Electron microscopy research of this family of receptors has determined that these receptors include seven transmembrane $\alpha$-helices. 
Moreover, each transmembrane helix has several specific residues and a common threedimensional structure that is conserved throughout all GPCRs (Lomize et al., 1999). The transmembrane protein structure contains seven stretches of 20-30 hydrophobic amino acids that form the membrane-spanning $\alpha$-helices. The protein has an extracellular $\mathrm{N}$-terminus and a cytoplasmic C-terminus. The N-termini of GPCRs varies greatly in length, ranging from seven amino acids for the adenosine receptor to over 300 amino acid residues for the glycoprotein hormone receptors. The $\mathrm{C}$-terminus has one phosphorylation site, which can influence signal transduction across the membrane. Furthermore, the C-terminal cysteine may be palmitoylated, through building an additional cytoplasmic loop, which may affect receptor mobility or G-protein coupling (Probst et al., 1992).

The ligand-binding sites of GPCRs have been partly described through both biochemical and molecular biological methods. Helical amino acids have been classified as (A) amino acids that are in association with membrane lipids and (B) amino acids that are not in association with membrane lipids (Baldwin, 1993). Amino acid residues that are conserved within GPCRs contribute to the specificity of ligand binding. For example, two conserved serines in transmembrane helix 5 of the $\beta 2$-adrenergic receptor (Ser204 and Ser207) have been involved in building hydrogen bonds with the meta- and para-hydroxyl groups of adrenergic agonists. In many hormone GPCRs, the Nterminus is glycosylated and is rich in cysteine residues that may form disulfide bridges and help maintain the three-dimensional structure of the protein. However, in the absence of the extracellular $\mathrm{N}$-terminus, ligands can still connect to the seven-transmembrane components of the receptor, albeit with a more inferior affinity (Ji and Ji, 1991). This suggests that GPCRs may include both a site extracellular binding with high affinity and a site in the within transmembrane domains of low affinity. It is possible that the site of the extracellular binding with high affinity created to capture the ligand and present it to the intramembranous binding pocket for signal transduction.

On the basis of this background, in the present study, we prepared the recombinant GPR161 receptor aiming to obtain monoclonal antibodies that can be used for developing diagnostics and therapeutics for TNBC. We generated a GPR161 fragment encompassing amino acids 64-344 (length 897 bp including restriction sites), cloned it into two different expression vectors, and transformed them into $E$. coli strain BL21 (DE3). We assessed and confirmed the expression of rTMGPR161 using one of the vectors, pET32/rTMGPR161, and optimized the conditions for the isolation and purification of rTMGPR161. Despite the many benefits of recombinant protein production in $E$. coli, this expression system has problems such as low expression levels or even lack of proteins expression (Soleimani et al., 2016). The lack of expression of rTMGPR161 in E. coli when using the $\mathrm{pET} 28$ vector is likely due to the formation of inclusion bodies.At the same time, the pET32 series is designed for cloning and high-level expression of peptide sequences fused with the thioredoxin protein. Thioredoxin significantly increases the solubility of proteins, while maintaining their biological activity (LaVallie et al., 1993).

We solubilized rTMGPR161 in a solubilization buffer and purified it by metalaffinity chromatography on a $\mathrm{Ni}^{2+}$-Sepharose column. As a result, a high initial concentration of the protein, which was effectively refolded while simultaneously being separated from highmolecular-weight protein aggregates, was obtained. Purification was achieved by spatially separating the rTMGPR161 molecules from each other through the pores of the column and a gradual increase in the concentration of imidazole. The LC-MS/MS analysis showed that rTMGPR161 was a fusion protein and contained thioredoxin and the TMGPR161 protein fragment.

The most widely used strategy to express GPCRs in E. coli is to use a fusion protein containing the receptor and a bacterial protein. The first example of this was the $\beta$ 2-adrenergic receptor, which had the first 279 residues of $\beta$ galactosidase, a cytosolic protein naturally expressed in E. coli, fused to its $\mathrm{N}$-terminus (Marullo et al., 1988). Fusion of the receptor to membrane proteins found in E. coli such as MBP or LamB increases the expression levels of the receptor by 10 -fold. Nevertheless, identical expression levels of the receptor can be obtained 
by using a strong IPTG-inducible promoter (gene 10 of the T7 bacteriophage) (Breyer et al., 1990; Nahmias et al., 1991). This difference clearly suggests that expression levels are receptordependent and that for each GPCR, a variety of genetic constructs must be regularly used because the results depend on the correct original sequence of the construct (Chapot et al., 1990).

\section{CONCLUSION}

In summary, we successfully expressed a recombinant form of GPR161, which we believe will be useful for the generation of either diagnostic or therapeutic monoclonal antibodies and can also be used as a protein therapeutic.

\section{ACKNOWLEDGEMENTS}

This research was performed within the framework of the budget program 217 of the Ministry of Education and Science, the Republic of Kazakhstan, on project No. AP05130053, "Obtaining monoclonal antibodies to GPR161 a marker of metastatic breast cancer," for the period 2018-2020. We would like to thank Editage (www.editage.com) for English language editing.

\section{REFERENCES}

Attrill, H., Harding, P. J., Smith, E., Ross, S., \& Watts, A. 2009. Improved yield of a ligand-binding GPCR expressed in $E$. coli for structural studies. Protein Expression and Purification 64: 32-38.

Baldwin, J. M. 1993. The probable arrangement of the helices in G protein-coupled receptors. EMBO Journal 12: 1693-1703.

Bradford, M. M. 1976. A rapid and sensitive method for the quantitation of microgram quantities of protein utilizing the principle of protein-dye binding. Analytical Biochemistry 72: 248-254.

Breyer, R. M., Strosberg, A. D., \& Guillet, J. G. 1990. Mutational analysis of ligand binding activity of beta 2 adrenergic receptor expressed in Escherichia coli. EMBO Journal 9: 26792684.

Chapot, M. P., Eshdat, Y., Marullo, S., Guillet, J. G., Charbit, A., Strosberg, A. D., \& Delavier-Klutchko, C. 1990. Localization and characterization of three different betaadrenergic receptors expressed in Escherichia coli. European Journal of Biochemistry 187: 137-144.
Feigin, M. E. 2013. Harnessing the genome for characterization of G-protein coupled receptors in cancer pathogenesis. FEBS Journal 280: 4729-4738.

Feigin, M. E., Xue, B., Hammell, M. C., \& Muthuswamy, S. K. 2014. G-protein-coupled receptor GPR161 is overexpressed in breast cancer and is a promoter of cell proliferation and invasion. Proceedings of the National Academy of Sciences 111: 4191-4196.

Foulkes, W. D., Smith, I. E., \& Reis-Filho, J. S. 2010. Triplenegative breast cancer. The New England Journal of Medicine 363: 1938-1948.

Haffty, B. G., Yang, Q., Reiss, M., Kearney, T., Higgins, S. A., Weidhaas, J., Harris, L., Hait, W., \& Toppmeyer, D. 2013. Locoregional relapse and distant metastasis in conservatively managed triple negative early-stage breast cancer. Journal of Clinical Oncology 24: 5652-5657.

Ji, I. H. \& Ji, T. H. 1991. Human choriogonadotropin binds to a lutropin receptor with essentially no $\mathrm{N}$-terminal extension and stimulates cAMP synthesis. The Journal of Biological Chemistry 266: 13076-13079.

Kreike, B., van Kouwenhove, M., Horlings, H., Weigelt, B., Peterse, H., Bartelink, H., \& van de Vijver, M. J. 2007. Gene expression profiling and histopathological characterization of triple- negative/basal-like breast carcinomas. Breast Cancer Research 9: 1-14.

Lachapelle, J. \& Foulkes, W. D. 2011. Triple-negative and basallike breast cancer: Implications for oncologists. Current Oncology 18: 161-164.

Laemmli, U. K. 1970. Cleavage of structural proteins during the assembly of the head of bacteriophage T4. Nature 227: 680685.

LaVallie E. R., DiBlasio E. A., Kovacic S., Grant K. L., Schendel P. F., \& McCoy J. M. 1993. A thioredoxin gene fusion expression system that circumvents inclusion body formation in the E. coli cytoplasm. Biotechnology 11 (2): 18793.

Lomize, A. L., Pogozheva, I. D., \& Mosberg, H. I. 1999. Structural organization of G-protein-coupled receptors. Journal of Computer-Aided Molecular Design 13: 325-353.

Marullo, S., Delavier-Klutchko, C., Eshdat, Y., Strosberg, A. D., \& Emorine, L. J. 1988. Human beta 2-adrenergic receptors expressed in Escherichia coli membranes retain their pharmacological properties. Proceedings of the National Academy of Sciences 85: 7551-7555.

Nahmias, C., Blin, N., Elalouf, J. M., Mattei, M. G., Strosberg, A. D., \& Emorine, L. J. 1991. Molecular characterization of the mouse beta 3-adrenergic receptor: Relationship with the atypical receptor of adipocytes. EMBO Journal 10: 3721 3727.

Probst, W. C., Snyder, L. A., Schuster, D. I., Brosius, J., \& Sealfon, S. C. 1992. Sequence alignment of the G-protein coupled receptor superfamily. DNA and Cell Biology 11:1-20.

Rakha, E. \& Reis-Filho, J. S. 2009. Basal-like breast carcinoma: From expression profiling to routine practice. Archives of Pathology \& Laboratory Medicine 133: 860-868.

Soleimani, M., Mirmohammad-Sadeghi H., Sadeghi-Aliabadi H., \& Jahanian-Najafabadi A. 2006. Expression and purification of toxic anti-breast cancer p28-NRC chimeric protein. Advanced Biomedical Research 5: 70-76.

Sotiriou, C. and Pusztai, L. 2009. Gene-expression signatures in breast cancer. The New England Journal of Medicine 360: 790800.

Towbin, H., Staehelin, T., \& Gordon, J. 1979. Electrophoretic transfer of proteins from polyacrylamide gels to nitrocellulose sheets: Procedure and some applications. Proceedings of the National Academy of Sciences 76: 4350-4354. 